\title{
Recognition of Letters and Letter Font in Images Using Deep Learning
}

\author{
Nilesh Korde ${ }^{* 1}$ and Abhijeet Ramesh Thakare ${ }^{2}$ \\ ${ }^{1,2}$ Shri Ramdeobaba College of Engineering and Management, Nagpur, India
}

\section{ABSTRACT}

The reason for this article is to perceive letters and particularly textual style from pictures which are containing writings. To perform the acknowledgment process, fundamentally, the content in the picture is separated into letters. At that point, each letter is sent to the acknowledgment framework. Results are separated by vowels which are generally utilized in English writings. Accordingly, the textual style of the content is gotten. To isolate letters from content, a calculation utilized which created detachment. This calculation is made for English letters which have specks. To give acknowledgment of English characters, all conceivable outcomes were made for every one of these characters, and the calculation was framed in like manner. In the wake of perceiving every letter, separate portions were submitted for processing to CNN system. The informational index contains about 13 a huge number of letters with $227^{*} 227^{*} 3$ size that have been made with various focuses, textual styles, and letters. Therefore, 100 percent of progress has been accomplished in the preparation. \% 79.08 letters and \%75 of text style achievement have been accomplished in the tests.

KEY WORDS: CONVOLUTION NEURAL SYSTEMS (CNN), LETTER ACKNOWLEDGMENT, PROFOUND LEARNING, TEXTUAL STYLE ACKNOWLEDGMENT.

\section{INTRODUCTION}

Lately, humankind is attempting to do all activities which are in everyday life, on advanced frameworks by diminishing and robotizing human force. This mechanization necessity empowered the formation of keen frameworks. Method of Profound learning used to re-enact the human mind 's structure. Used technique depicts progression of calculations for searching various leveled portrayal information via mimicking how that individual cerebrum faculties significant pieces of a lot of tangible information that it is presented to consistently. The thought originated with the premise

\section{ARTICLE INFORMATION}

Received 10th Oct 2020 Accepted after revision 29th Dec 2020 Print ISSN: 0974-6455 Online ISSN: 2321-4007 CODEN: BBRCBA

Thomson Reuters ISI Web of Science Clarivate Analytics USA and Crossref Indexed Journal

\section{Clarivate
Analytics}

NAAS Journal Score 2020 (4.31) SJIF: 2020 (7.728)

A Society of Science and Nature Publication,

Bhopal India 2020. All rights reserved.

Online Contents Available at: http//www.bbrc.in/

Doi: http://dx.doi.org/10.21786/bbrc/13.14/20 of profound wisdom developed in the year 1950 based on the perception definition. Perception has the capacity to learn. The recognition to perception structure specially multilayer received during the period of 1980s. Be that as it may, the perception has constrained learning capacity. Hence, the proposition of neural systems with numerous layers developed during the 2000s.

The structure alongside this proposal has been exceptionally equipped for knowledge. The numerous levels of profound wisdom foundation. CNN systems, specially concentrates on the picture, demonstrating elite and accomplishments, also identified to be profound wisdom structure that conveys improved outcomes. This model helpful for finding designs in pictures to perceive items, appearances, and scenes. They gain legitimately from picture information, utilizing examples to arrange pictures and disposing of the requirement component removal physically. This model consisting of multilevel structure, every level containing a majority of twodimensional planes and a majority of neurons each plane.

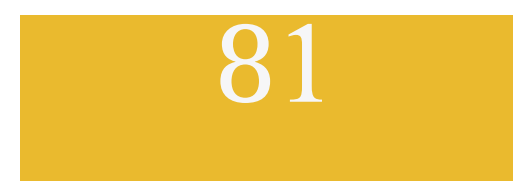


Furthermore, within old-style AI, the appropriate response is just 1 or 0 , and in the yield of the investigations utilizing this system structure, values somewhere in the range of 0 and 1 , for example, 0.2 and 0.7 can be acquired. This makes it simpler to tackle the issue in a progressively itemized manner, it expands the accomplishment in learning and gives better outcomes. In the wake of learning highlights in numerous layers, the following piece of a convolutional neural system is grouping. The CNN system design utilizes a grouping levels for giving the characterization yield. This paper has been divided into five segments. The main segment of this paper gives concise information on profound learning. The subsequent area surveys the related works. The third area is worried about the system utilized for this investigation. The fourth segment shows the discoveries of the examination. At long last, we give the end.

Related Works: Lately, considerably more data has gotten accessible on profound learning. Thusly a lot of writing has been distributed on this subject. Regardless of whether convolutional neural systems have been enhanced during the long period of time, prevalence of it has picked from many years. The numerous levels of profound wisdom foundation. Expanding the information, some unsolved issues dependent on picture and film preparing, its deficiency of existing knowledge strategies expanded the notoriety of profound perception. Thus, a large portion of the investigations taking place considering the effectiveness of CNN systems. These days, with extremely thriving capacities, profound deep networks being utilized in support of order, recognition, conclusion. Letter acknowledgment in digitized records is significant for chronicling. Some digitized archives contain two pictures and content. So, a simple neural network processor designed and actualized for visual letter acknowledgment during 1991.

During examination, manually written letters with 20X20 size have been utilized. In one more examination, optical substance recognizable proof was acknowledged with the neural system. Chinese character acknowledgment was acknowledged by utilizing different convolutional neural systems. Textual style acknowledgment was considered by certain scientists. Character free textual style acknowledgment on a solitary Chinese character was examined. Since expressed in given article, acknowledgment exactness was expanded using quantity of the letters utilized for acknowledgment. An additional investigation done on Arabic text style acknowledgment. The anticipated framework was founded on manageable geometry and indicated the elite.

\section{MATERIAL AND METHODS}

In the vast majority of the investigations, written by hand letter acknowledgment has been executed for various dialects. An examination was discovered, that two measurements optical letter acknowledgment dependent on profound learning, underpins English letters. No text style acknowledgment study, for English, was found in the writing. In this examination, it is expected to perceive the textual style of the content in a picture and to separate the letter and text style data in the picture. To the extent we inquire about, this is the primary investigation on textual styles that help English letters in the applications up until this point. Fundamentally we perceived the characters along with the text styles of the characters. Thus system of character acknowledgment comprises English script. The third division is worried regarding the technique of picture handling prior to the picture is sent to organize. The fourth division clarifies the manner for utilizing the character partitioning. The last division illustrates the outcomes.

Data Set: As no readied informational collection was establish in the writing, another informational collection was made for this investigation. In the initial segment, the informational index was arranged by characters, and the later division information was sequenced using text styles. Within letter arrangement, pictures were made for every one of the 29 letters. Just letters were incorporated, digits were prohibited during investigation. Since pictures might include various sizes of letters which were chosen. Including these lines, three letter pictures were made as large, medium and little for every lower and capitalized letter. Focused on keeping 72 points as large, 20 points as medium and 8 points as little.

Once the arrangement of all the letter pictures were made, pictures were resized as $271^{*} 271$ for a profound convolution neural system. Then, 228 letter pictures for single letter and 6612 letter pictures for the entire letters in order were made. Another informational index has been made by choosing 38 text styles that help English letters and utilizing these textual styles in every one of the 29 letters in the English letters in order. All shapes and size cases were incorporated and a sum of 38 textual styles were utilized for single letter and 174 letter pictures for single text style, and 6612 letter pictures for the sum total of what text styles were made altogether. Subsequently, the arrangement part has been finished. Some of the models from the database are given below.

\section{Figure 1: Models from Dataset}

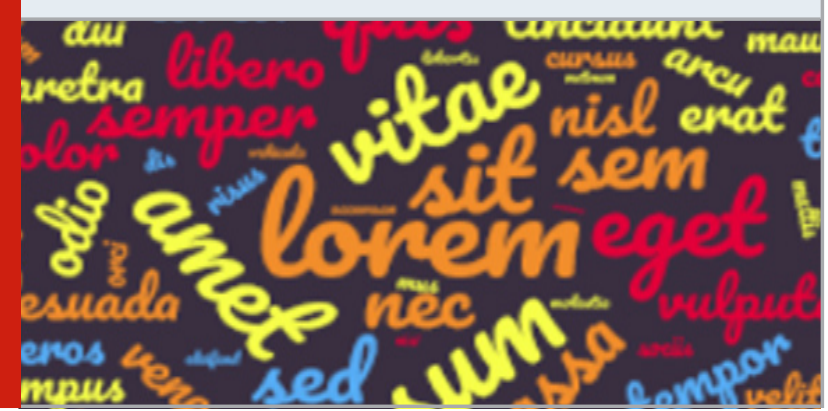

Creation of Profound Learning System: During examination, Alex net was utilized to make neural system which generally remembered for the profound learning tool compartment of Mat lab $\odot$. Alex net was prepared to classify thousand of various types of pictures and it was prepared on various other million pictures. Consisting about 25 layers, the greater part among 
layers were doing helpful picture prepartion that would work for our framework. Firstly, the preparation pictures were appeared in the system. Furthermore, information would split into preparing and test pictures. overall 80 percent pictures were decided for preparing pictures by randomizing. later on, the pre-prepared system, Alex Net, would be changed. The 23rd layer of Alex Net consisting a thousand neurons as Alex Net characterizes a thousand diverse images. It was adjusted to 29 for the primary system along with 38 for the second. In the wake of setting up all system parameters, the preparation procedure was figured out. In the wake of preparation done, the system was tried. Since the exactness was acceptable, the system was spared.

\section{Pre-processing of Picture}

The picture was prepared before it was sent to the system. These activities were recorded beneath.

- In the initial step, the picture was changed over to the power picture group.

- In the second step, the picture was changed over to a twofold organization.

- In the third step, the picture was changed over to supplement itself, as a result of morphological picture preparing.

- In the last advance, morphologic picture preparation was utilized for finding every one of letter areas. We have utilized a capacity that profits the name network that contains marks for the 8-associated (even, vertical and corner to corner) questions in picture

Figure 2: Preparing Process Plot

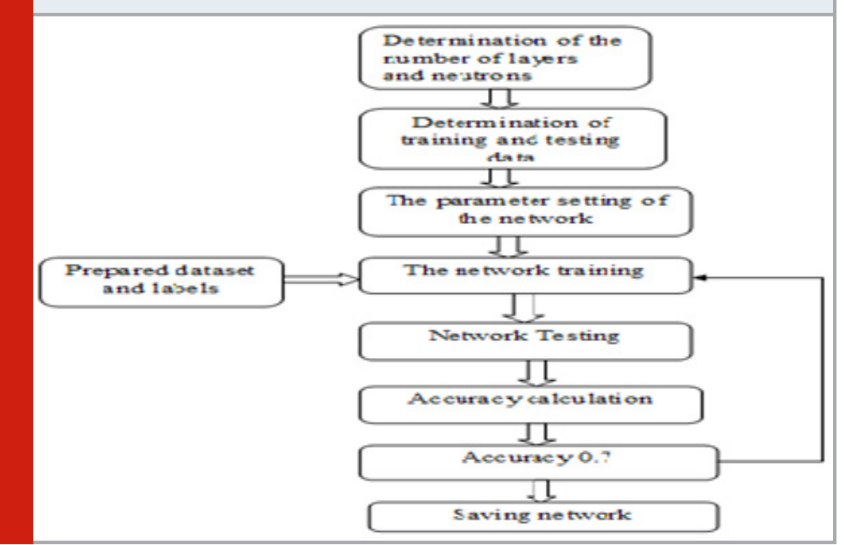

Figure 3: Pre-processing Plan

Sit $\rightarrow$ Sit $\rightarrow \begin{aligned} & 1010101010 \\ & 0101110010 \\ & 1000011101 \\ & 1001110101\end{aligned} \quad \rightarrow$ SE匹 $>$ Sit

Separation of Letter: In the wake of pre-processing steps that were done, letters were isolated and found by marking the picture. The total of what steps were recorded underneath.

- Corner directions of every single marked field was established.

- It was concluded that this marked field was a letter or part of a letter by making relative estimations.

- If the letter would be a complemented letter, for example, "g" or dabbed letter, for example, "i, j, ü or ö", the directions of the marked field were refreshed by these pieces of letters.

- As per calculation, there were three conceivable outcomes. Within the principal cycle if the calculation was demonstrated when first field was a piece of a letter and the subsequent field was a letter then this was the "I" letter. Else If the first field was a letter and the subsequent field was a part of the letter then this would be " $j$ or g" letters. Else if the primary field was a letter and the second and third fields were portions of a letter then this would be the "ü or ö" letter. If one of the conceivable outcomes becomes genuine, at that point update the directions. Once the directions of the entire letters including English letters were characterized, the picture was changed over to a simulated rub position once more, just for the system necessity.

- $\quad$ Size was scaled to $271^{*} 271$.

- Each letter was sent to the prepared letter arrange. As indicated by the system yield mark, the related letter was added to the content.

- Once every letter acknowledgment, every letter was sent to the textual style organized. As indicated by the system yield name, related text style name was spared.

- After every letter textual style was accomplished separately, it was seen that the system result for every letter can some of the time be extraordinary. Even though the content of the test picture was novel textual style, every one of the letter textual styles can be various because of the trouble of textual style acknowledgment. So we conquer this trouble with a likelihood count. The outcome text style was achieved with likelihood.

Printout the Outcomes: The outcomes have appeared in two sections. The initial segment is preparing results with subsequent division is acknowledgment outcomes. The accompanying tables demonstrate the preparation outcomes. To expand the exactness of the textual style acknowledgment, the outcomes have been approved by most utilized English characters. For approving the outcomes, after being discovered every character and every text styles of the letters, its being tallied to each character textual style recurrence. The outcome textual style of the picture is concluded by most elevated recurrence. Specified system being prepared for 38 distinct textual styles. The system has been tried with 12 test pictures in which their properties given in Table III. Lorem Ipsum pictures made for testing. Table I and Table II depict training results. Test outcomes have been given in Table IV.

\section{RESULTS AND CONCLUSION}

The investigation expecting for building up a profound system, perceiving the two text styles and characters in English. Nearly 13 thousand images have been prepared for the pre-prepared system. The character 
acknowledgment preparing precision was \%100, and text style acknowledgment preparing exactness was $\% 73.44$, in light of the closeness of the textual styles. In any case, to build the textual style acknowledgment rate, a probability estimation has been utilized, after the system yield has been found. Regardless of whether the main test picture text style exactness is $14 / 26$, on account of the probability is greater than 0.5 , its being acknowledged as Arial. So with along these lines, the acknowledgment execution expanded more. The system tried with 12 pictures. Pictures contain only characters. As indicated by the outcomes, character acknowledgment in this system expected about \%100 rate, however, precision about textual style acknowledgment is low, as it can clearly seen from Table IV. Be that as it may, utilizing the likelihood, textual style acknowledgment rate being expanded.

Figure 4: Stream Graph of Acknowledgment

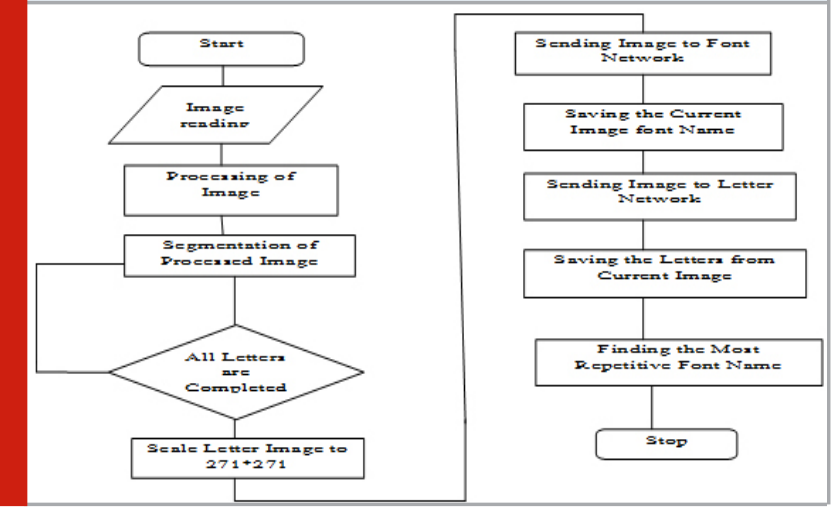

Table- I: Training Textual Style System Results
\begin{tabular}{|c|c|c|c|c|c|}
\hline Epoch & Iteration & $\begin{array}{c}\text { Time } \\
\text { Elapsed }\end{array}$ & $\begin{array}{c}\text { Mini- } \\
\text { batch } \\
\text { Accuracy }\end{array}$ & $\begin{array}{c}\text { Mini- } \\
\text { batch } \\
\text { Loss }\end{array}$ & $\begin{array}{c}\text { Base } \\
\text { Learning } \\
\text { Rate }\end{array}$ \\
\hline 1 & 1 & $00: 00: 01$ & $1.58 \%$ & 4.3096 & 0.0010 \\
\hline 2 & 150 & $00: 06: 34$ & $18.20 \%$ & 2.4671 & 0.0010 \\
\hline 3 & 200 & $00: 08: 13$ & $30.15 \%$ & 2.3336 & 0.0010 \\
\hline 4 & 250 & $00: 14: 48$ & $36.55 \%$ & 1.8929 & 0.0010 \\
\hline 5 & 500 & $00: 19: 45$ & $40.08 \%$ & 1.7260 & 0.0010 \\
\hline 6 & 650 & $00: 23: 02$ & $51.03 \%$ & 1.5559 & 0.0010 \\
\hline 7 & 800 & $00: 27: 58$ & $44.78 \%$ & 1.4569 & 0.0010 \\
\hline 8 & 1050 & $00: 32: 55$ & $61.93 \%$ & 1.2811 & 0.0010 \\
\hline 9 & 1300 & $00: 44: 26$ & $64.61 \%$ & 0.8712 & 0.0010 \\
\hline 10 & 1600 & $00: 56: 41$ & $72.45 \%$ & 0.8155 & 0.0010 \\
\hline
\end{tabular}

\begin{tabular}{|l} 
Table- I: Training Letter System Results \\
\begin{tabular}{|c|c|c|c|c|c|}
\hline Epoch & Iteration & $\begin{array}{c}\text { Time } \\
\text { Elapsed }\end{array}$ & $\begin{array}{c}\text { Mini- } \\
\text { batth } \\
\text { Accuracy }\end{array}$ & $\begin{array}{c}\text { Mini- } \\
\text { batch } \\
\text { Loss }\end{array}$ & $\begin{array}{c}\text { Base } \\
\text { Learning } \\
\text { Rate }\end{array}$ \\
\hline 1 & 1 & $00: 00: 01$ & $3.13 \%$ & 3.4619 & 0.0010 \\
\hline 2 & 150 & $00: 05: 58$ & $91.63 \%$ & 0.1500 & 0.0010 \\
\hline 3 & 300 & $00: 11: 55$ & $94.31 \%$ & 0.4740 & 0.0010 \\
\hline 4 & 550 & $00: 17: 52$ & $100.00 \%$ & 0.1866 & 0.0010 \\
\hline 5 & 700 & $00: 23: 49$ & $98.44 \%$ & 0.0094 & 0.0010 \\
\hline 6 & 850 & $00: 29: 46$ & $100.00 \%$ & 0.0151 & 0.0010 \\
\hline 7 & 1000 & $00: 35: 43$ & $97.44 \%$ & 0.0217 & 0.0010 \\
\hline 8 & 1250 & $00: 41: 40$ & $100.00 \%$ & 0.02165 & 0.0010 \\
\hline 9 & 1600 & $00: 47: 43$ & $98.44 \%$ & 0.0034 & 0.0010 \\
\hline 10 & 1900 & $00: 57: 53$ & $100.00 \%$ & 0.0078 & 0.0010 \\
\hline
\end{tabular}
\end{tabular}

ACKNOWLEDGEMENTS

The authors would like to thank the Department of Computer Science and Engineering and Department of Computer Application at Shri Ramdeobaba College of

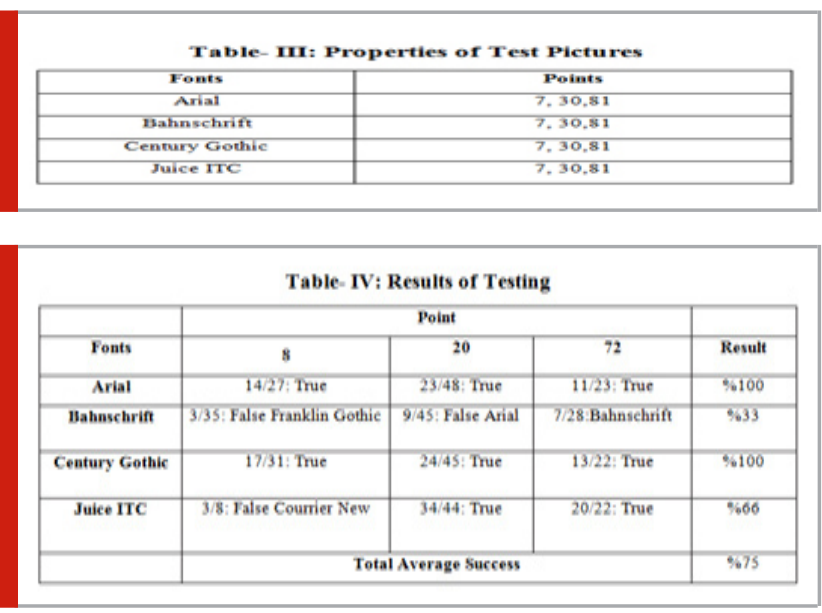

Engineering and Management Nagpur.

\section{REFERENCES}

A.Koyun, E. Afsin, ”2D optical character acknowledgment dependent on profound learning," Diary of Turkey Informatics Establishment of Software engineering and Designing, 2017, vol. 10, no. 1, pp. 11-14.

A.Riley, P. Work and R. Mill operator, "Visual element recognizable proof: a neural system approach," IJCNN91-Seattle Global Joint Gathering on Neural Systems, Seattle, WA, USA, 1991, pp. 909 vol. 2.

E. Bati, "Profound convolution neural systems with an application towards geospatial object acknowledgment," Diss. Center East Specialized College Ankara, 2014.

M. U. Oner, "Metastasis discovery and limitation in lypmh hubs by utilizing convolutional neural systems," Diss. Center East Specialized College.

M. Oquab, L. Bottou, I. Laptev and J. Sivic, "Learning and moving mid-level picture portrayals utilizing convolutional neural systems," 2014 IEEE Gathering on PC Vision and Example Acknowledgment, Columbus, Gracious, pp. 1717-1724.

P. Kuang, W. Cao and Q. Wu, "See on structures and calculations of profound learning," 2014 eleventh Global PC Gathering on Wavelet Actiev Media Innovation and Data Processig (ICCWAMTIP), Chengdu, 2014, pp. 176179.

Q. - . Wu, Y. L. Cun, L. D. Jackel and B. - . Jeng, ”On-line acknowledgment of restricted jargon Chinese character utilizing different convolutional neural systems," 1993 IEEE Global Symposium on Circuits and Frameworks, Chicago, IL, 1993, pp. 2435-2438 vol.4.

R. Wason, "Profound learning: Development and extension," Intellectual Framework Exploration, 2018, vol. 52, pp.701-708.

Z. Hu, J. Tang, Z. Wang, K. Zhang, L. Zhang and Q. Sun, "Profound learning for picture based disease identification and analysis An overview," Example 
Acknowledgment, vol. 83, 2018, pp. 134-149, ISSN. 0031-3203.

X. Ding, L. Chen, T. Wu, "Character autonomous textual style acknowledgment on a solitary chinese character," in IEEE Exchanges on Example Investigation and Machine Intelligence,2007, vol. 29, no. 2, pp. 195-
204.

F. K. Jaiem, F. Slimane, M. Kherallah, ”Arabic textual style acknowledgment framework applied to various content substance level anlysis," 2017 Worldwide Meeting on Brilliant, Checked and Controlled Urban areas (SM2C), Sfax, 2017, pp. 36-40. 